\title{
A GENERALIZATION OF A THEOREM OF NEHARI
}

\author{
BY R. P. GILBERT ${ }^{1}$ AND H. C. HOWARD ${ }^{1}$
}

Communicated by Walter Rudin, July 23, 1965

In 1956 Nehari, [N.1] showed that the singular points $t_{0}$ of the Legendre series,

$$
\phi(t)=\sum_{n=0}^{\infty} a_{n} P_{n}(t), \quad|t+1|+|t-1|<\rho+\frac{1}{\rho},
$$

where $\lim \sup _{n \rightarrow \infty}\left|a_{n}\right|^{1 / n}=\rho^{-1}<1$, are related to the singular points $\zeta_{0}$ of the associated power series,

$$
f(\zeta)=\sum_{n=0}^{\infty} a_{n} \zeta^{n}, \quad|\zeta|<\rho^{-1}
$$

by the formula $t_{0}=\frac{1}{2}\left(\zeta_{0}+1 / \zeta_{0}\right)$, providing $t_{0} \neq \pm 1$. The purpose of this note is to announce similar results concerning the singularities of functions $\phi(z)$ defined by series of the form $\sum_{n=0}^{\infty} a_{n} v_{n}(z)$, where the $v_{n}(z)$ are normalized eigenfunctions of the Sturm-Liouville system

$$
\begin{aligned}
v^{\prime \prime}(z)+\left(\rho^{2}-q(z)\right) v(z) & =0, \\
v^{\prime}(0)+h v(0)=v^{\prime}(\pi)+H v(\pi) & =0 .
\end{aligned}
$$

Indeed we are able to establish the following result.

THEOREM. Let $q(z) \in C^{\infty}[0, \pi]$, let the $v_{n}(z)$ be the set of normalized eigenfunctions of the Sturm-Liouville system (3), and let $\left\{a_{n}\right\}$ be a sequence of complex numbers such that $\lim \sup _{n \rightarrow \infty}\left|a_{n}\right|^{1 / n}=\rho^{-1}<1$. Furthermore, let us introduce the pair of analytic function elements defined in a neighborhood of the origin,

$$
\begin{gathered}
f(\zeta)=\sum_{n=0}^{\infty} a_{n} \zeta^{n}, \quad|\zeta|<\rho^{-1} \\
\psi(t)=\sum_{n=0}^{\infty} a_{n} u_{n}(t), \quad|t+1|+|t-1|<\rho+\frac{1}{\rho}
\end{gathered}
$$

1 This research was supported at the institute for Fluid Dynamics and Applied Mathematics of the University of Maryland in part by the National Science Foundation under Grants GP-2067 and GP-3937, and in part by the Air Force Office of Scientific Research under Grant AFOSR 400-64, and at Georgetown University by the National Science Foundation under Grant GP-5023. 
where $u_{n}(\cos z) \equiv v_{n}(z)$. Then, providing $t \neq \pm 1$, the function $\psi(t)$, defined above is singular at those points $t=\frac{1}{2}(\alpha+1 / \alpha)$, where $\zeta=\alpha$ is a singular point of the series $f(\zeta)$.

Sketch of Proof. The method of proof parallels that given originally by Nehari [N.1] which has been extended by Gilbert [G.1-4], and Gilbert and Howard [G.H.1-2], who have used these ideas in conjunction with Bergman's integral operator method [B.1-2] for the study of partial differential equations. (See also the survey paper by Gilbert, Howard and Aks [G.H.A.1].)

We are able to introduce an integral operator $\mathcal{F}[f]$, which maps power series (A) onto the eigenfunction series (B). Furthermore, we also are able to construct an inverse operator $\mathfrak{F}^{-1}[\psi]$, which maps $\psi(t)$ onto $f(\zeta)$. These operators may be seen to have the form given below,

$$
F[f] \equiv \int_{|\zeta|-\rho_{0}} K(t, \zeta) f(\zeta) \frac{d \zeta}{\zeta},
$$

where $1<\rho_{0}<\rho$, and

$$
\mathfrak{F}^{-1}[\psi] \equiv \int_{-1}^{+1} K\left(t, \zeta^{-1}\right) \psi(t) d t
$$

where the integration is along the real axis, and where $K(t, \zeta)$ $\equiv \sum_{n=0}^{\infty} u_{n}(t) \zeta^{-n}$. By the use of the elementary Hartogs' theorem [B.M.1], and known appraisals for the $v_{n}(z)$ (and hence for the $\left.u_{n}(t)\right)$ as $n \rightarrow \infty$ we are able to establish that $K\left(t, \zeta^{-1}\right)$ is a holomorphic function of two complex variables in certain product domains; consequently, we may consider the above integrals as Cauchy integrals.

We are also able to determine the first analytic set (moving outwards from the origin in the $\zeta$-plane) on which $K\left(t, \zeta^{-1}\right)$ is singular, as given by $\left\{\zeta^{2}-2 \zeta t+1=0\right\}$. Using this information plus the argument used by Hadamard [H.1], [N.1] in his proof of the "multiplication of singularities" theorem, allows us to establish the fact that if $f(\zeta)$ is singular at $\zeta=\alpha,(|\alpha|=1 / \rho)$, then in the compact ellipse, $|t+1|+|t-1| \leqq \rho+1 / \rho, \psi(t)$ is regular for all points $t \neq \frac{1}{2}(\alpha+1 / \alpha)$. Correspondingly, we are able to show if $\psi(t)$ has a singularity on the boundary of the above ellipse, say at $t=\sigma,\left(\sigma=\frac{1}{2}(\alpha+1 / \alpha)\right)$, then $f(\zeta)$ is regular at all points $\zeta \neq \alpha$, such that $|\zeta| \leqq|\alpha|$. Combining these results yields the above theorem.

REMARK. We have also been able to find analogous results for eigenfunction expansions associated with quite general $n$th order SturmLiouville systems. These results along with the details of the above proof will be published elsewhere. 


\section{REFERENCES}

B.1. S. Bergman, Integral operators in the theory of linear partial differential equations, Ergebnisse der Mathematik und Grenzgebiete, N. F. No. 23, Springer, Berlin, 1961.

B.2. - Application of integral operators to the theory of singular partial differential equations and computation of compressible fluid flow, Symposium on Numerical Analysis, Univ. of Maryland, College Park, Md., 1965.

B.M.1. S. Bochner and W. T. Martin, Several complex variables, Princeton Univ. Press, Princeton, N. J., 1948.

G.1. R. Gilbert, Singularities of three-dimensional harmonic functions, Pacific J. Math. (4) 10 (1960), 1243-1255.

G.2. - On harmonic functions of four variables with rational $P_{4}$-associates, Pacific J. Math. (1) 13 (1963), 79-96.

G.3. - Bergman's integral operator method in generalized axially symmetric potential theory, J. Math. Phys. 5 (1964), 983-997.

G.4. - Multivalued harmonic functions of four variables, J. Analyse Math. 15 (1965), 305-323.

G.H.1. R. Gilbert and H. C. Howard, On solutions of the generalized bi-axially symmetric Helmholtz equaiion, Proc. London Math. Soc. (3) 15 (1965), 346-360.

G.H.2. - Integral operator methods for generalized axially symmetric potentials in ( $n+1)$-variables, J. Austral. Math. Soc. (to appear).

G.H.A.1. R. Gilbert, H. Howard, and S. Aks, Singularities of analytic functions having integral representations with a remark about the elastic unitarity integral, J. Math. Phys. (7) 6 (1965), 1157-1162.

H.1. J. Hadamard, Theoreme sur les series entières, Acta Math. 22 (1898), 55-64.

N.1. Z. Nehari, On the singularities of Legendre expansions, J. Rational Mech. Anal. 5 (1956), 987-991.

GEORGETOWN UNIVERSITY AND

UNIVERSITY OF WISCONSIN-MILWAUKEE 